\title{
Renal Artery Embolism as Differential Diagnosis of Nephrocolicae \\ Suzana $\mathrm{K}^{1}$, Bernard $\mathrm{B}^{1}$, Gordana $\mathrm{D}^{1}$, Helga $\mathrm{SM}^{2}$, Marina $\mathbf{S}^{1}$ and Cuti EC ${ }^{3^{*}}$ \\ ${ }^{1}$ Clinic for Pulmonary Diseases, University Hospital Centre, Jordanovac, Zagreb, Croatia \\ ${ }^{2}$ Clinical Department for Diagnostic and Interventional Radiology, University Hospital Center, Zagreb, Croatia \\ ${ }^{3}$ Department of Internal Medicine, Zabok General Hospital, Zabok, Croatia
}

*Corresponding author: Edina Catic Cuti, Department of Internal Medicine, Zabok General Hospital, Bracak 8, 49210 , Zabok, Croatia, Tel: +385 49 204 000; E-mail: edina3110@gmail.com

Received date: March 09, 2016; Accepted date: Dec 06, 2017; Published date: Dec 12, 2017

Copyright: (C) 2017 Suzana K, et al. This is an open-access article distributed under the terms of the Creative Commons Attribution License, which permits unrestricted use, distribution, and reproduction in any medium, provided the original author and source are credited.

\begin{abstract}
Renal artery embolism was described first in year 1940, but recently it is recognized as a clinically significant entity. It is a rare event, mostly seen in patients of 60 to 80 years old, with certain predisposing risk factors for tromboembolism, dominantly with underlying cardiovascular disease including atrial fibrillation, rheumatic heart disease, cardiac heart failure, arterial hypertension and general atherosclerosis. Renal artery embolism causes asymptomatic to sudden onset of nonspecific symptoms such as fever, abdominal pain, vomiting and nausea. There are few treatment options for renal artery embolism and some of them are early anticoagulation therapy and trombolytic therapy with streptokinase or tissue plasminogen activator preferably in early stage $90-180$ minutes from onset. Medicamentous can be choice as a first line of treatment is more favourable than surgical therapy that is preferred in patients with solitary kidney or bilateral emboli.
\end{abstract}

Keywords: Renal artery embolism; Thromboembolism; Case Report

Atherosclerosis; Arterial hypertension; Cardiac heart failure

\section{Introduction}

Renal artery embolism is a rare event, mostly seen in patients 60 to 80 years old, with certain predisposing risk factors for tromboembolism, dominantly with underlying cardiovascular disease including atrial fibrillation, rheumatic heart disease, cardiac heart failure, arterial hypertension and general atherosclerosis [1-5].

Clinical presentation vary from being asymptomatic to sudden onset of nonspecific symptoms such as fever, abdominal pain, vomiting and nausea $[1,2,4,5]$. Due to the fact it can mimic nephrolythiasis or is without any symptoms, there is a higher risk of delayed or missed diagnosis [1,2]. Also, unusual presentation such as lower extremity motor weakness accompanying abdominal pain was reported by Akdur et al. [6]. Laboratory parameters, serum lactate dehydrogenase $(\mathrm{LDH})$ as a sensitive enzyme marker, leucocytes, creatinin value and aspartate transaminase (AST) considered as nonspecific marker but helpful are usually elevated [7]. Haematuria in urine analysis is also the most common finding. Interestingly, according to the literature in most reported cases right kidney was involed with no clear etiology [5].

Contrast enhanced CT is considered to be a gold standard for diagnosis of renal artery embolism in the emergency department $[1,2,3,5]$

Treatment options are early anticoagulation therapy and trombolytic therapy with streptokinase or tissue plasminogen activator preferably in early stage 90-180 minutes from onset [4,5]. Medicamentous choice as a first line of treatment is more favourable than surgical therapy that is preferred in patients with solitary kidney or bilateral emboli [5,7].
We present a case of a 74 year old woman with a history of arterial hypertension, metabolic syndrome, paroxysmal atrial fibrillation, coronary disease and general atherosclerosis presented at the Emergency Department (ED) due to fever and cough starting one week before as well as with chest pain and dyspnea on minimal usual exertion during the previous month, which intensified in the days preceding the admission. The patient's relevant medical history included a myocardial infarction 20 years earlier and a PCI with endovascular prosthesis implantation 8 years earlier. It is important to note that the patient was not under regular supervision of a cardiologist and last control visit was seven years ago, also she did not take prescribed products regulary. The therapy she was using (with no precise information about last revision) was amiodarone, bisoprolol, clopidogrel, acetylsalycylic acid, furosemide with potassium product, simvastatin, lisinopril in combination with hydrochlorothiazide and ranitidine.

On physical examination, the patient was conscious, pale, subfebrile $\left(37.4^{\circ} \mathrm{C}\right)$, dyspneic, orthopneic with respiratory rate 25 breaths $/ \mathrm{min}$, blood pressure $185 / 100 \mathrm{mmHg}$ and heart rate $80 / \mathrm{min}$, with left lung crepitation on auscultation, slightly enlarged liver, peripheral oedema and symmetrically slightly weaker peripheral arterial pulsation.

Laboratory tests showed an elevation of the inflammatory parameters, leucocytes $\left(10.3 \times 10^{9} / \mathrm{L}\right)$, C reactive protein $(68.08 \mathrm{mg} / \mathrm{L})$ and a mild increase of troponin $(0.035 \mu \mathrm{g} / \mathrm{L})$ in scope of the systemic inflammatory response due to pneumonia and congestive heart failure. The electrocardiogram showed sinus rhythm with a stable rate $80 / \mathrm{min}$ and no changes in the ST segment, an acute coronary event was ruled out.

The patient was hospitalized due to clinically and radiologically verified acute heart failure and pneumonia. Initial treatment including loop diuretic furosemide, an antibiotic and oxygen therapy led to a 
significant clinical improvement within the following few days. On the third day of hospitalization, the patient reported a new onset right hemiabdominal pain with nausea, vomiting and a single loose stool. On clinical examination, the abdomen was soft, elastic with no organomegaly, but with right hemiabdominal tenderness and audible peristalsis.

Initial urgent laboratory findings including $\mathrm{LDH}$, serum amylase, serum creatinine and liver transaminase values were unremarkable and radiological findings revealed a few smaller air-fluid levels in the projection of the ascending colon. Urine analysis was not preformed. An antiemetic and an antispasmodic were added to the therapy. In the next few hours, a deterioration of the clinical status was observed with persistant dull right hemiabdominal pain, predominantly under the right costal arch and along the right lateral wall, now also involving the right lumbar region which was tender on percussion. The repeated laboratory tests showed an increase in serum creatinine $(187 \mu \mathrm{mol} / \mathrm{l})$, liver transaminase (AST $214 \mathrm{U} / \mathrm{L})$ and LDH (1241 U/L).

An emergency MSCT of the abdomen and pelvis (Figures 1 and 2) was performed, revealing an outbreak of the right kidney opacification in the arterial and venous circulation phases, with an embolus in the area of the right renal artery origin protruding eccentrically toward the abdominal aortic lumen. It measured approximately $10 \mathrm{~mm}$ in diameter and had visible and more abundant marginal sclerotic plaques in the area of origin of both renal arteries. There was no evidence of blood flow through the right renal artery. However, another smaller segmental branch for the lower pole of the right kidney was visible with a maintained flow. The blood flow through renal veins and the inferior vena cava was maintained as well as the blood flow through the right common and external iliac veins.

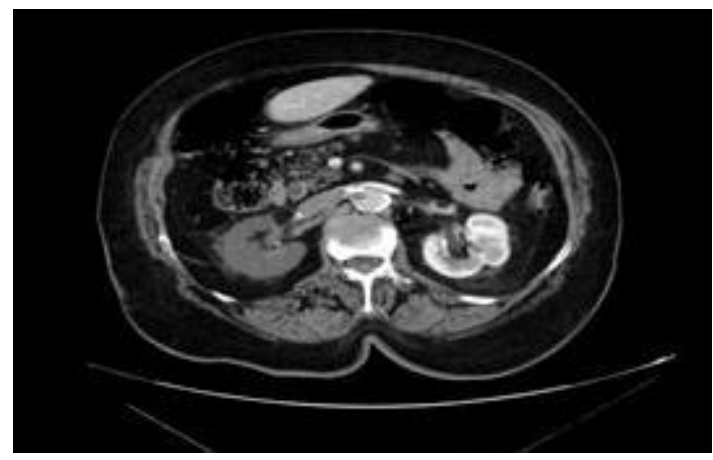

Figure 1: Coronal recon computed tomography enhanced with IV contrast exhibiting an irregular hypodense- The right kidney consistent with hypo-perfusion, Normal perfusion- The left kidney.

The echocardiogram showed a regular systolic function with ejection fraction of $55 \%$ with no verified valvular vegetations or pericardial effusion. In line with the diagnosis, anticoagulation therapy with low molecular weight heparin in therapeutic dosage was initiated. Thrombolysis was not a therapy of choice due to patient's age and risk of bleeding and the time from first symptoms onset was longer than 180 minutes. In the later course of treatment a moderate increase of nitric substances was observed without tendency to progression.

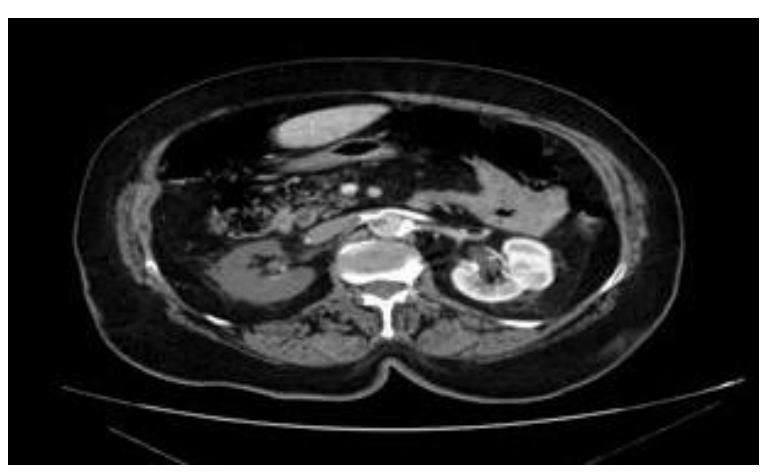

Figure 2: Contrast enhanced axial CTA image of abdomen shows expansile clot within the right main renal artery which bulges into the aortic lumen itself and a decreased perfusion of the right kidney.

The patient received parenteral hydration along with diuretics in order to eliminate contrast medium applied during angiography. Adequate diuresis was observed subsequently, without renal insufficiency progression and without need for hemodialysis. In the later course the patient achieved an overall good recovery.

\section{Conclusion}

Renal artery embolism is an important clinical entity in the emergency department due to the fact it can lead to irreversible loss of renal function and is life threatening condition. That is why we want to remind us all that we should consider renal artery embolism as a differential diagnosis in patients with known cardiovascular disease and other risk factors, presenting with abdominal pain, accompanying vomiting, nausea and laboratory elevated levels of AST, LDH and serum creatinine. Due to diagnostic and treatment advances of today's medicine, it can be successfully managed with overall good recovery and perserved renal function.

\section{References}

1. Tan TW, Bohannon WT, Mattos MA, Hodgson KJ, Farber A (2011) Percutaneous mechanical thrombectomy and pharmacologic thrombolysis for renal artery embolism: Case report and review of endovascular treatment. I J Angiol 20: 111-116.

2. Baydar O, Baskurt M, Coskun U, Ersanli M (2013) A case of renal artery embolism treated by selective intra-arterial infusion of tissue plasminogen activator. Turk Kardiyol Dern Ars 41: 534-536.

3. Kansal S, Feldman M, Cooksey S, Patel S (2008) Renal artery embolism. J Gen Intern Med 23: 644-647.

4. Cheng KL, Tseng SS, Tarng DC (2003) Acute renal failure caused by unilateral renal artery thromboembolism. Nephrol Dial Transplant 18: 833-835.

5. Chu PL, Wei YF, Huang JW, Chen SI, Chu TS, et al. (2006) Clinical characteristics of patients with segmental renal infarction. Nephrology 11: 336-340.

6. Akdur O, Durukan P, Ozkan S, Ikizceli I, Avsarogullari L, et al. (2008) Renal artery embolism in a patient with vague abdominal pain. Letter to the Editor 37.

7. Uta H, Michael K, Hermann P, Martin H, Eckhart B (2008) A rare case of acute renal failure- acute bilateral renal artery ambolism. Nephrol Dial Transplant 23: 2095-2097. 